\title{
Dynamic Lithium Distribution Upon Dendrite Growth and Shorting Revealed by Operando Neutron Imaging
}

\author{
Bohang Song ${ }^{\dagger}, *$, Indu Dhiman ${ }^{\dagger}, *$, John C. Carothers ${ }^{\dagger}$, Gabriel M. Veith ${ }^{\ddagger}$, Jue Liu ${ }^{\dagger}$, Hassina Z. \\ Bilheux ${ }^{\dagger}$, and Ashfia Huq ${ }^{\dagger}$ \\ $\dagger$ Neutron Scattering Division, Oak Ridge National Laboratory, Oak Ridge, Tennessee 37831, \\ United States \\ $\$$ Material Science and Technology Division, Oak Ridge National Laboratory, Oak Ridge, \\ Tennessee 37831, United States \\ * Correspondence: bohangsong@gmail.com, id.indudhiman@gmail.com
}




\section{Contents}

S1. Experimental methods.

S2. Normalization of the transmission neutron radiographs

S3. Depth effect of Li concentration gradient in cathode electrode

S4. Detailed description on the evolutional average transmission intensity.

Table S1. Elemental neutron cross sections $\left(1 \mathrm{barn}=10^{-24} \mathrm{~cm}^{2}\right)$ for a neutron wavelength of 2.6 Å.

Figure S1. SEM (original (left) and zoomed-in area (right)) images of the thick electrode shows the spatial relationship between the aluminum framework and the composite island, where the composite island contains $\mathrm{LiMn}_{2} \mathrm{O}_{4}$ spherical particles.

Figure S2. Picture of Li metal after cycling with a thick electrode.

Figure S3. Evolution of representative Li-volumes in cathode electrode at different stages of charging.

Figure S4. In situ normalized neutron radiographs of electrochemical cell measured while discharging.

Figure S5. Average transmission intensity for different region of interests (ROIs) to depict removal of $\mathrm{Li}$ as a function of time at different stages of discharging.

Video S1. Videos of normalized neutron radiographs as a function of time during the whole charging/discharging cycle.

Video S2. (a) 3D rotation video of the cell at the pristine state, (b) translation through the cell exhibiting the Li distribution at the pristine state.

Video S3. (a) 3D rotation video of the cell at the fully-charged state, (b) translation through the cell exhibiting the Li distribution at the fully-charged state.

Video S4. (a) 3D rotation video of the cell at the fully-discharged state, (b) translation through the cell exhibiting the Li distribution at the fully-discharged state.

References 


\section{S1. Experimental methods}

\section{Design of the in situ electrochemical cell}

We have developed an in situ electrochemical cell that can be used to perform neutron radiography and computed tomography experiments. This cell has been demonstrated for a successful neutron scattering experiment during battery cycling. ${ }^{1}$ The schematic of the electrochemical cell is shown in Scheme 1(a) in the main text. The core part of this cell design is a thick working electrode (cathode) that enables massive Li transportation. This is essentially important for the neutron radiography measurements due to its limited resolution $(\sim 100 \mu \mathrm{m})$. The preparation method of thick electrode is explained in our previous report. ${ }^{1}$ Natural Li was used to synthesize $\mathrm{LiMn}_{2} \mathrm{O}_{4}(200 \mathrm{mg})$ to prepare the cathode electrode ( $8 \mathrm{~mm}$ thick), which also contained carbon black (37 mg), polyvinylidene fluoride $(37 \mathrm{mg})$, and Al metal $(150 \mathrm{mg}) .{ }^{7} \mathrm{Li}$ (ORNL National Isotope Development Center, $99.92 \%$ ) and glass fiber was used as the counter electrode and separator to enhance the contrast between the deposited natural-Li and the ${ }^{7} \mathrm{Li}$ electrode when dendrites form. Deuterated electrolyte (d-ethylene carbonate and d-dimethyl carbonate containing $\operatorname{LiPF}_{6}(1 \mathrm{M})$ in a 3:7 volume ratio) was used to reduce the incoherent scattering of hydrogen.

\section{Operando neutron imaging experiment}

Neutron radiography and tomography experiments with cold neutrons were performed at the CG-1D imaging beamline, at the High Flux Isotope Reactor (HFIR), Oak Ridge National Laboratory. ${ }^{2}$ The schematic of experimental set-up is depicted in Scheme 1(b).

Neutron attenuation contrast to map Li distribution was achieved with a charge coupled detector (CCD) camera system (iKon - L 936, Andor Technology plc. Belfast, UK) using a 50 
$\mu \mathrm{m}$ thick $\mathrm{LiF} / \mathrm{ZnS}$ scintillator. The field of view (FOV) for this set-up was $7.4 \times 7.4 \mathrm{~cm}^{2}$, with the effective pixel size of $36 \times 36 \mu \mathrm{m}^{2}$. The measurements were carried out using a polychromatic cold neutron beam having a wavelength range from 0.8 to $6 \AA$, with a peak intensity of $2.2 \times 10^{6} \mathrm{n} / \mathrm{cm}^{2} / \mathrm{s}$ at $2.6 \AA$. With the detector-to-pinhole distance of $\mathrm{L}=6.59 \mathrm{~m}$, and a pinhole size $\mathrm{D}=16 \mathrm{~mm}$, the obtained spatial resolution was $\sim 100 \mu \mathrm{m}$ (with an $\mathrm{L} / \mathrm{D}$ ratio of $\sim$ 410).

Table S1 lists the neutron absorption cross sections $\left(1 \mathrm{barn}=10^{-24} \mathrm{~cm}^{2}\right)$ of all the possible elements that are exposed to the neutron beam, at a wavelength $\lambda=2.6 \AA$. Note that natural $\mathrm{Li}$ has the highest absorption cross section compared to other elements present in the cell, which makes it possible to map the Li distribution in this experiment. The charge/discharge profiles of the battery during the in situ experiment and the correlation with the radiography/tomography measurements performed is shown in Scheme 1(c). The cell was charged at a rate of C/50 (current is $0.48 \mathrm{~mA}$, where $1 \mathrm{C}=120 \mathrm{~mA} / \mathrm{g}$ ), implying $50 \mathrm{~h}$ for fully charging the battery. Such a low rate guarantees enough $\mathrm{Li}$ transportation between the cathode and the anode. Initially, while charging the battery, neutron radiographs were collected continuously for $30 \mathrm{~s}$ each (with a dead time of $\sim 3.8 \mathrm{~s}$ that is needed to send each radiograph from the detector to the local computer) using the CCD camera system. After charging for approximately $10 \mathrm{~h}$ at each stage (the first charging costs $6 \mathrm{~h}$ ), tomography scans (approximately $12 \mathrm{~h}$ acquisition time) were performed during the battery resting (i. e. battery not being charged). During the third charging process, the massive dendrite growth caused the battery shorting internally, but the battery was still charged until $\sim 31.5 \mathrm{~h}$. Thereafter, the battery was set to discharge $(0.48 \mathrm{~mA}$ for $8 \mathrm{~h}$ and $0.96 \mathrm{~mA}$ for the rest) and simultaneously neutron radiographs were collected. Finally, a tomography measurement was carried out on a completely discharged battery. Using three consecutive 
charging is to make sure the dendrite grows significantly to short the battery, in the meantime, the tomography of the cell could be built up as a function of charging time. Each data set was reconstructed using commercially available OCTOPUS software package with the standard filtered back-projection algorithm. ${ }^{3}$ For further analysis, commercially available AMIRA $^{4}$ software was used for the 3D visualization of the electrochemical cell, qualitative assessment of Li transport and deposition, and to estimate the representative Li-volume within the cathode electrode. In addition, the median filter feature of AMIRA was utilized to offer the most visual clarity of the images. The intensity range of the voxels in all the reconstructed volume shown was adjusted in such a way that the major contribution from $\mathrm{Li}$ is retained, while the other parts of the electrochemical cell were rendered transparent.

\section{S2. Normalization of the transmission neutron radiographs}

All the transmission radiographs measured as a function of time were normalized using the first neutron radiograph at the pristine state. This way only contribution from Li migration is visible and the contribution for all the other components of the cell is removed.

\section{S3. Depth effect of Li concentration gradient in cathode electrode}

Figure S3a depicts a representative Li-volume as a function of depth in the working electrode at different stages of charging in order to investigate an inhomogeneous (de)lithiation reactions. To extract the value of representative Li-volume, the 3D reconstructed neutron tomography data sets were segmented using interactive thresholding tool available in AMIRA software. Initially, using the pristine state data, we obtained one threshold value of the intensity to segment the Li present in the working electrode. This threshold value was kept fixed for all the other 3D datasets. Thereafter, to obtain the depth profile, the regions of interest (ROI) with a same volume as a 
function of electrode depth from different states of charging were selected. Then, the value of representative Li-volume for each ROI was extracted using the threshold described above. Note that this representative $\mathrm{Li}$-volume is not an accurate volume of $\mathrm{Li}$ in the working electrode due to a complex attenuation from all elements under the neutron beam. However, it is good enough to represent the changing trend when the concentration of $\mathrm{Li}$ in a volumetric $\mathrm{ROI}$ varies as a result of (de)lithiation reactions.

In Figure S3a, at the pristine state the depth profile of the representative Li-volume exhibits an upward trend; this is attributed to the electrode preparation method, which induces an increasing amount of $\mathrm{LiMn}_{2} \mathrm{O}_{4}$ particles along the depth. Similar behavior is depicted at different stages of charging, with representative Li-volume increasing along the depth. It is worthwhile pointing out that the threshold chosen in recognition of the representative Li-volume is based on the pristine state, which implies that the concentration is one $\mathrm{Li}$ atom per $\mathrm{LiMn}_{2} \mathrm{O}_{4}$ formula. Thus, the volume of $\mathrm{Li}$ roughly represents the volume of $\mathrm{LiMn}_{2} \mathrm{O}_{4}$ particles. During charging, the particles with overall $\mathrm{Li}_{1-\mathrm{x}} \mathrm{Mn}_{2} \mathrm{O}_{4}$ composition $(0<\mathrm{x}<1)$ cannot account for the contribution of the representative Li-volume in individual regions of interest (ROIs). In this regard, the gaps (marked by different levels of darkness) between two curves of the representative Li-volume at different charging steps (Figure S3a), for instance between the pristine and the first charging step, depicts the volume of $\mathrm{LiMn}_{2} \mathrm{O}_{4}$ particles that are actively delithiated at an individual depth, not necessarily fully delithiated. Figure S3b displays how the cathode was arbitrarily sectioned into three layers (top, middle and bottom). The inset of Figure S3a plots the values of these gaps between two charging steps for the top and the bottom layer individually. It is seen that in the bottom layer the gap significantly increases as the charging proceeds, while in the top layer this gap gradually reduces. It indicates that $\mathrm{Li}$ is first depleted from the top layer of the cathode and 
then gradually depleted towards the bottom layer of the electrode during charging. A depth effect of the delithiation reaction when the electrode is thick is clearly demonstrated. Figures S3b and S3c provide a visualization evidence of how much $\mathrm{LiMn}_{2} \mathrm{O}_{4}$ volume reacted along the depth of the cathode as a function of charging time. Apparently, the $\mathrm{LiMn}_{2} \mathrm{O}_{4}$ volume in the top layer reduces significantly from the pristine to the first charging state, while the counterpart in the bottom layer has almost no change. In contrast, both layers show significantly reduced volumes of $\mathrm{LiMn}_{2} \mathrm{O}_{4}$ from the first to the third charging state.

\section{S4. Detailed description on the evolutional average transmission intensity}

During the first charging step (Figure 3(a) in the main text), the average transmission intensity of the top layer cathode exhibits an upward trend as a function of charging time. While for the other ROIs below the top layer no significant change is observed, indicating that the depletion of Li begins from the top most layer of the cathode. During the second charging process, the lower depth ROIs also begin to show an increase in transmission intensity. Though in comparison with the top most layer the increase in transmission intensity is not so drastic. The above observation of the depth effect on the delithiation of electrode agree well with the 3D evidence (Figure S3). During the third charging step shown in Figure 3(c) (main text), initially up to approximately 24 $\mathrm{h}$, for all the ROIs Li continues to migrate out of the cathode. At approximately $24 \mathrm{~h}$, the battery shorts. As a result, the transmission intensity begins to exhibit overall downward trend, decreasing with an increased charging time for most of the ROIs.

In contrast, the trend of the dendritic-Li ROIs is due to Li accumulation and dendrite growth, consequently lowering the intensity, as shown in Figure 3(d-f) (main text). During the first charging step, except for the top most layer, all other ROIs exhibit decreasing trend of transmission intensity as a function of charging time. It implies that while charging the depleted 
Li from the top most layer of the cathode accumulates at the separator/Li-metal interface first and thereafter grows towards the cathode side. Therefore, the decline slope of each ROIs with the increase of charging time increases from the Li-metal towards the cathode side, correlated with the various growing rate of Li dendrite in different ROIs. Similar trend is observed for the rest of the charging steps (Figure 3(e-f) in the main text) before approximately $24 \mathrm{~h}$. Note that the top most ROIs layer of dendritic-Li region is within the Li metal electrode, as a result no change in transmission intensity is observed. The discharging step reverses this process to a significant degree (see Figure S5 in supporting information). This results in each ROIs experiencing an increasing trend in the overall transmission intensity. 
Table S1. Elemental neutron cross sections $\left(1 \mathrm{barn}=10^{-24} \mathrm{~cm}^{2}\right)$ for a neutron wavelength of 2.6 A.

\begin{tabular}{|c|c|c|c|c|c|c|c|c|c|c|c|}
\hline Elements & $\mathrm{Li}$ & ${ }^{7} \mathrm{Li}$ & $\mathrm{Mn}$ & $\mathrm{O}$ & $\mathrm{C}$ & $\mathrm{Al}$ & $\mathrm{Ti}$ & $\mathrm{Zr}$ & $\mathrm{Si}$ & $\mathrm{P}$ & $\mathrm{F}$ \\
\hline $\begin{array}{c}\text { Neutron } \\
\text { absorption cross } \\
\text { sections }\end{array}$ & 101.9 & 0.066 & 19.232 & 0.000 & 0.005 & 0.334 & 8.806 & 0.268 & 0.247 & 0.249 & 0.014 \\
\hline $\begin{array}{c}\text { Neutron } \\
\text { coherent cross } \\
\text { sections }\end{array}$ & 0.454 & 0.619 & 1.750 & 4.232 & 5.551 & 1.495 & 1.485 & 6.440 & 2.163 & 3.307 & 4.017 \\
\hline $\begin{array}{c}\text { Neutron } \\
\text { incoherent cross } \\
\text { sections }\end{array}$ & 0.920 & 0.780 & 0.400 & 0.000 & 0.001 & 0.008 & 2.780 & 0.020 & 0.004 & 0.005 & 0.001 \\
\hline
\end{tabular}



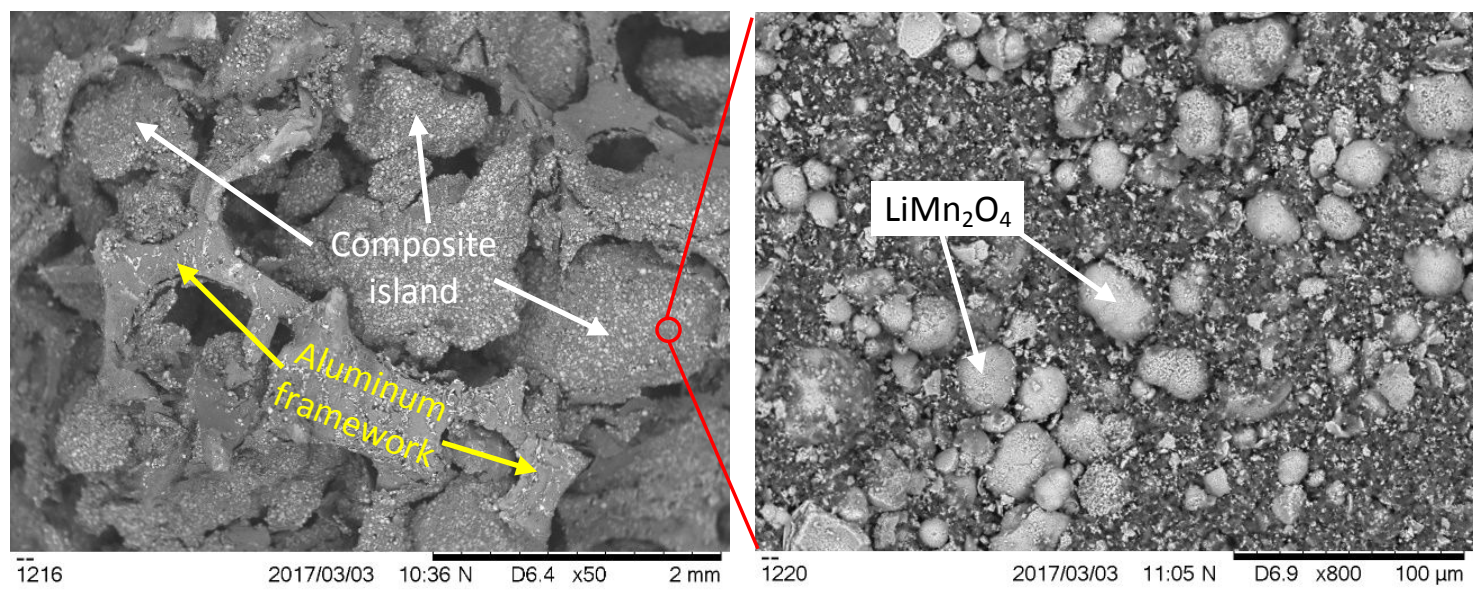

Figure S1. SEM (original (left) and zoomed-in area (right)) images of the thick electrode shows the spatial relationship between the aluminum framework and the composite island, where the composite island contains $\mathrm{LiMn}_{2} \mathrm{O}_{4}$ spherical particles. 


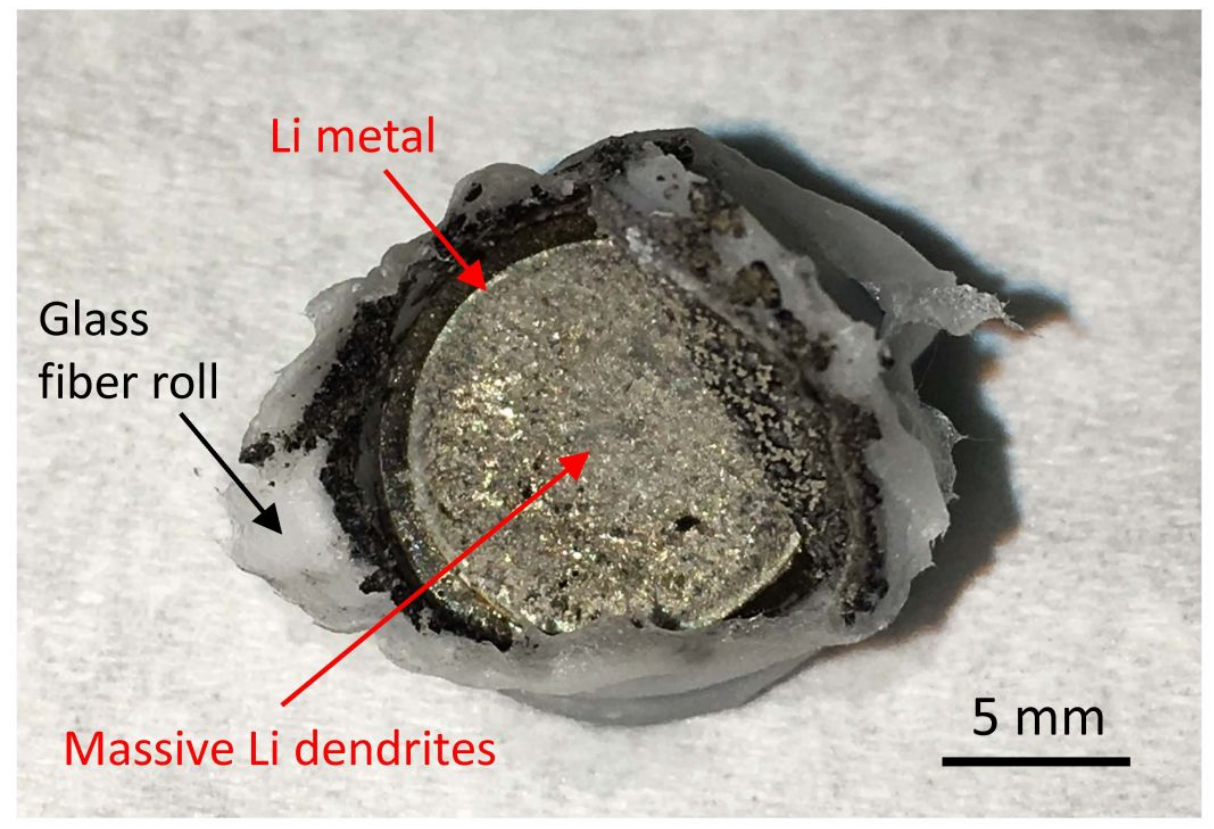

Figure S2. Picture of Li metal electrode after cycling with a thick cathode. 
(a)

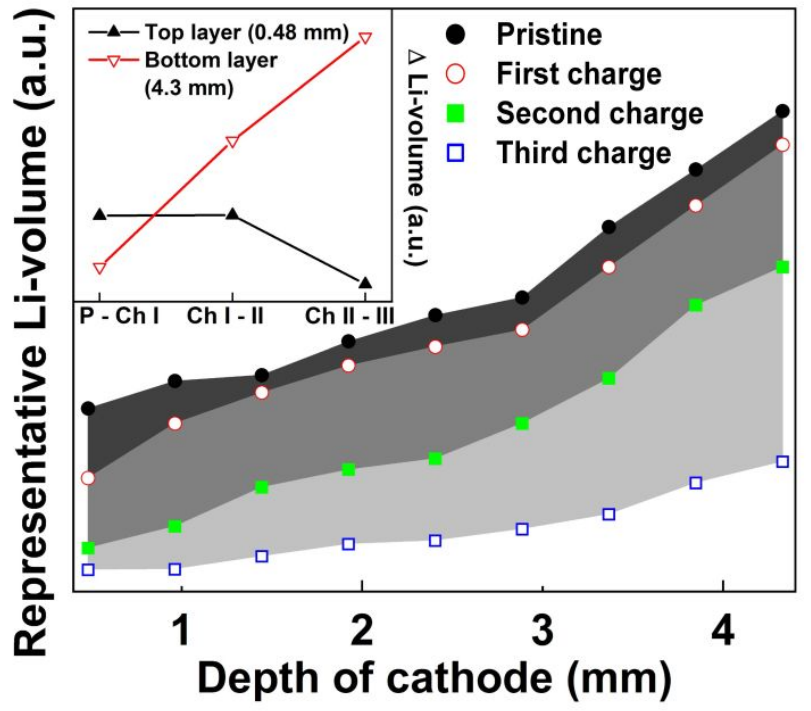

(c)

Top

layer

Middle

layer

Bottom

layer
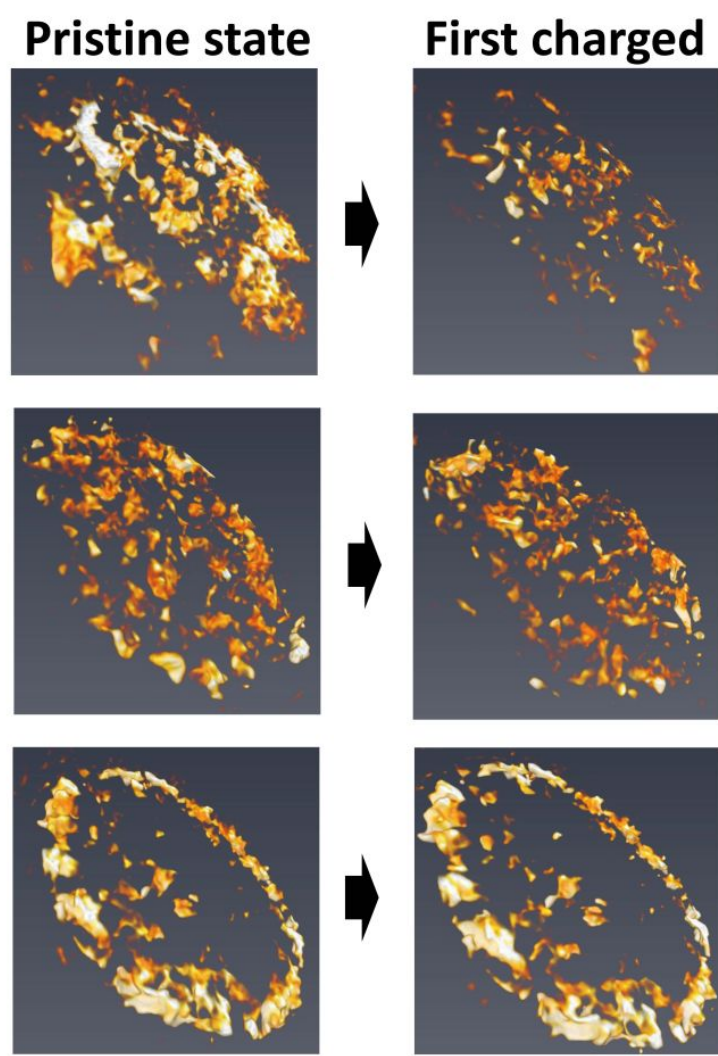

(b)

3D-view of battery
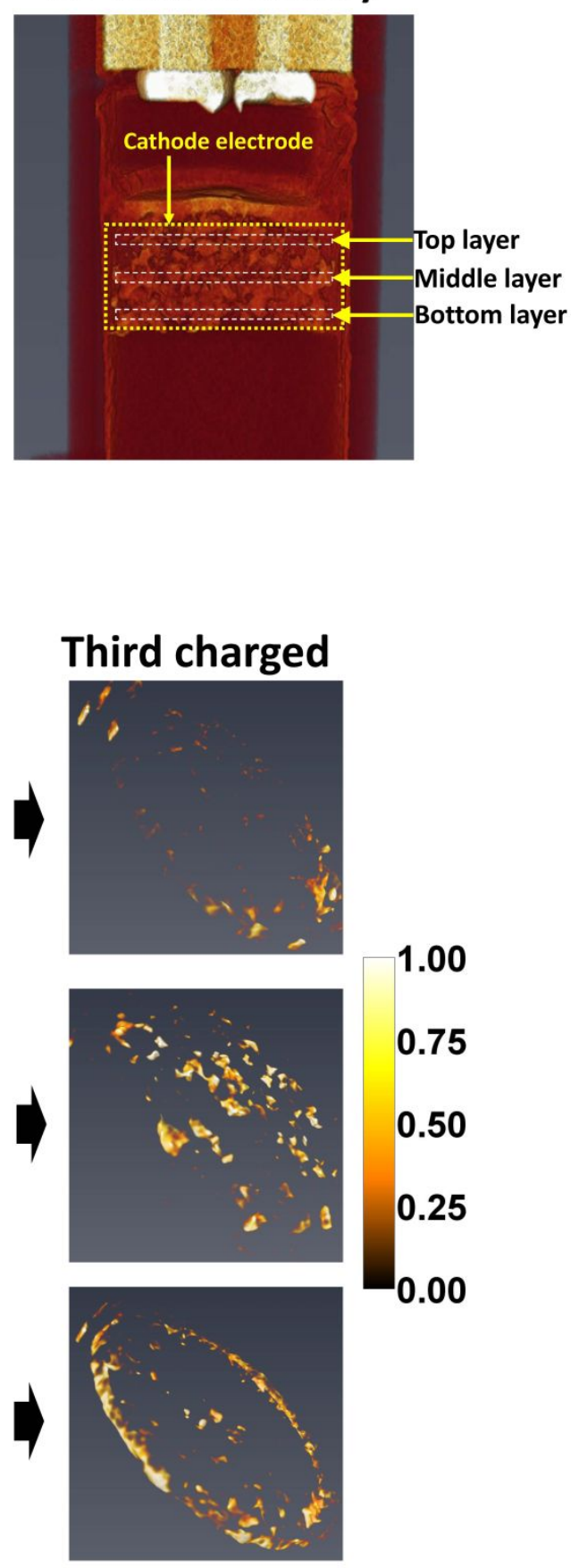

Figure S3. Evolution of representative Li-volumes in cathode electrode at different stages of charging. (a) Plots of representative Li-volume, (b) 3D-view of the battery showing the ROIs, and (c) the 3D reconstruction of the representative Li-volume of each ROI as a function of depth 
in the cathode electrode. Refer to the detailed explanation on the meaning of representative Livolume in S3 of the Supplementary Information. The inset of (a) depicts the change in the representative Li-volume in the top and bottom most layer of the cathode electrode, where it shows that in the bottom layer the reacted amount of $\mathrm{LiMn}_{2} \mathrm{O}_{4}$ particles increases while in the top layer the amount reduces as charging proceeds. "P" and "Ch" refer to the pristine and the charge state. The volume of $\mathrm{Li}$ (yellow regions) in (c) stands for the volume of fully-lithiated $\mathrm{LiMn}_{2} \mathrm{O}_{4}$ particles. 


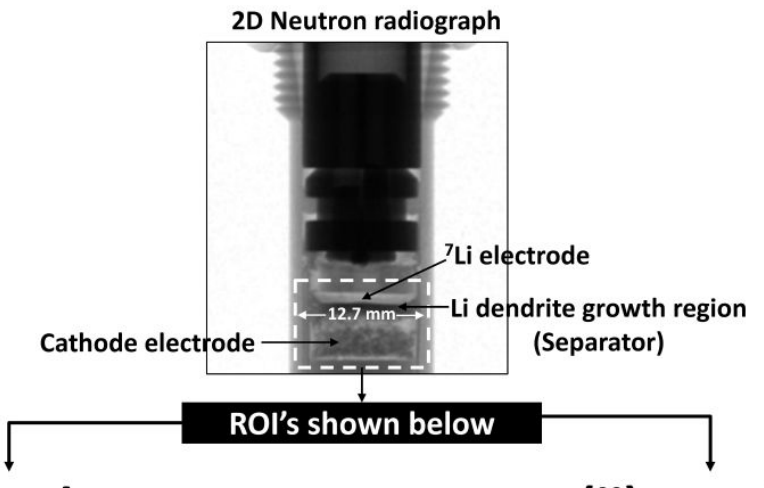

(i) $34.7 \mathrm{hrs}$

(ii) $39.1 \mathrm{hrs}$
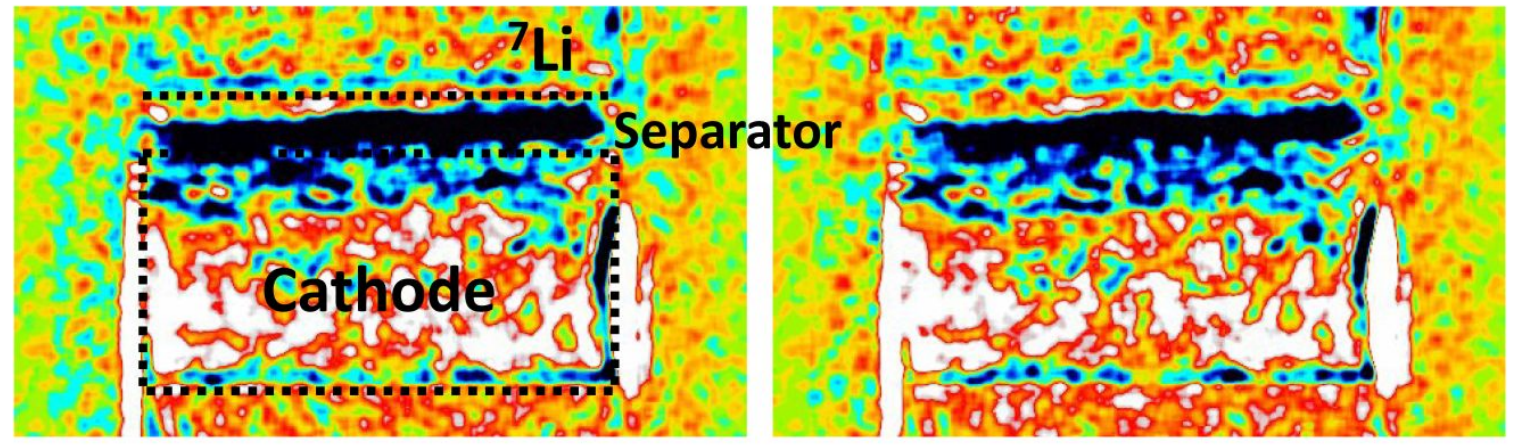

Figure S4. In situ normalized neutron radiographs of electrochemical cell measured while discharging. Migration of Li back to the cathode with considerable inhomogeneity is evidenced. 

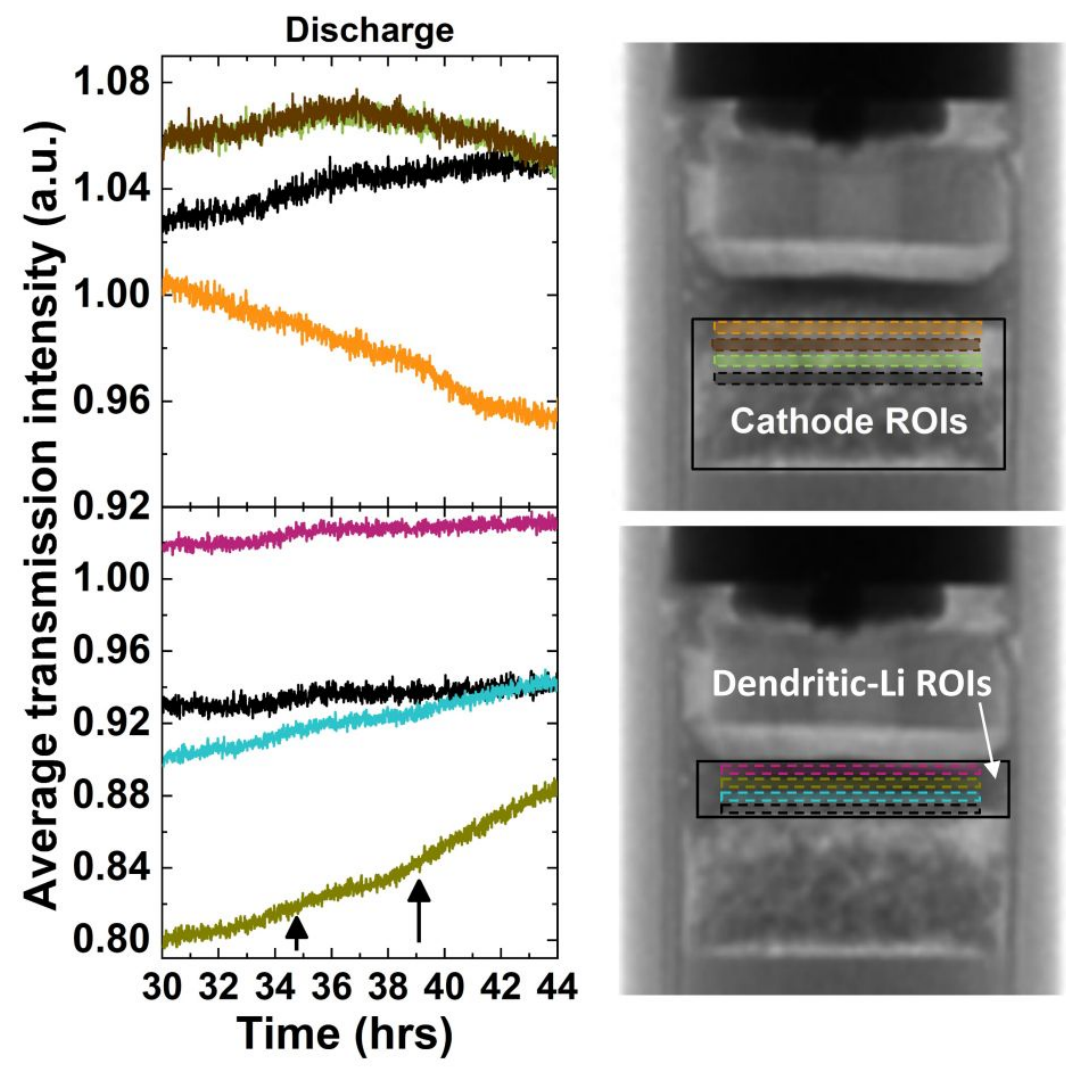

\section{Dendritic-Li ROIs}

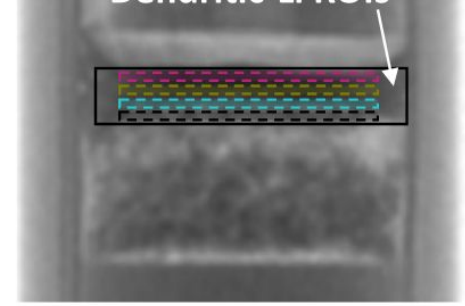

Figure S5. Average transmission intensity for different region of interests (ROIs) to depict removal of $\mathrm{Li}$ as a function of time at different stages of discharging. The correlated ROIs are shown in the neutron radiographs on the right. Arrows indicate the neutron radiographs shown in Figure S2. Error bars in the figure are smaller than the fluctuations in the line symbols. 


\section{References}

1. Song, B. H., Veith, M. G., Park, J., Yoon, M., Whitfield, S. P., Kirkham, M., Liu, J., Huq, A. (2018). Metastable $\mathrm{Li}_{1+\delta} \mathrm{Mn}_{2} \mathrm{O}_{4}(0 \leq \delta \leq 1)$ spinel phases revealed by in operando neutron diffraction and first-principles calculations. Chem. Mater. 31, 124-134.

2. Santodonato, L., Bilheux, H., Bailey, B., Bilheux, J., Nguyen, P., Tremsin, A., Selby, D., Walker, L. (2015). The CG-1D neutron imaging beamline at the Oak Ridge National Laboratory High Flux Isotope Reactor. Physcs. Proc. 69, 104-108.

3. Kak, A. C., Slaney, M. (1988). Principles of computerized tomographic imaging (IEEE Press).

4. Detlev Stalling, D., Malte Westerhoff, M., Hege, H.-C. (2005). Amira: a highly interactive system for visual data analysis (THE VISUALIZATION HANDBOOK). 